\title{
The incremental priming technique: A method for determining within-condition priming effects
}

\author{
ARTHUR M. JACOBS \\ Cognitive Neuroscience Laboratory, Brain \& Language Group \\ Centre National de la Recherche Scientifique, Marseille, France \\ and \\ JONATHAN GRAINGER and LUDOVIC FERRAND \\ Laboratoire de Psychologie Expérimentale \\ Centre National de la Recherche Scientifique, Université René Descartes, Paris, France
}

\begin{abstract}
A novel priming technique is applied in two experiments using an alphabetic decision and a lexical decision task to study effects of repetition, and form-related priming on letter and word recognition. The incremental priming technique consists of a gradual increase of the prime's informational value (operationalized as prime intensity). The minimum prime-intensity level serves as a withincondition baseline for each priming condition. Thus, we can define any priming effect with respect to two baseline conditions: one is the minimum-intensity condition of the particular priming condition (within-condition baseline), and the other is a different priming condition (across-condition baseline). This double-baseline approach makes measuring of priming effects more reliable and imposes stronger constraints on our interpretations of these effects.
\end{abstract}

The questions concerning the nature of the mental codes involved in reading and their time course of activation and decay have occupied cognitive psychologists for many years. The standard assumption today is that the system processing written language automatically computes multiple codes representing the input stimuli and that these codes have identifiable functional properties (Carr, 1986). Indeed, most current models of pattern recognition in general and letter and word recognition in particular assume that a visual stimulus is processed by activating a cascade of levels of representation that compute internal codes of different degrees of abstractness. For example, current models in the tradition of Selfridge's pandemonium model for pattern recognition (Selfridge \& Neisser, 1960), such as the interactive activation model (McClelland \& Rumelhart, 1981), its semistochastic and noninteractive variants (Jacobs \& Grainger, 1992), or the activation-verification model (Paap, Newsome, McDonald, \& Schvaneveldt, 1982), assume that the presentation of letter stimuli activates a set of feature detectors, which activate compatible letter detectors. The letter detectors, in turn, activate higher level units in the mental lexicon and in the response decision system compatible with them (for review see Jacobs \& Grainger, 1994). Recent evi-

We wish to thank Max Coltheart, Ken Forster, Lester Krueger, Ken Paap, and Tom Sanocki for their valuable comments on earlier versions of this paper. Part of this work was presented in Jacobs, Grainger, and Ferrand (1994) at the 35th Annual Meeting of the Psychonomic Society, St. Louis. Correspondence should be addressed to A. M. Jacobs, Brain \& Language Group, CNRS-LNC, 31 Chemin Joseph-Aiguier, F-13402 Marseille Cedex 20, France (e-mail: jacobs@lnf.cnrs-mrs.fr). dence from studies using brain imaging techniques supports this standard assumption (Posner \& Carr, 1992).

A convenient way of studying the nature of these hypothetical mental codes is to use priming techniques. A particular priming technique has recently attracted the attention of researchers in the domain of reading because it is supposed to prevent subjects from employing response strategies by using very short, masked primes (Ferrand \& Grainger, 1992; Forster \& Davis, 1984; Grainger \& Ferrand, 1994; Grainger \& Jacobs, 1991, 1993a; Humphreys, Evett, \& Quinlan, 1990; Jacobs \& Grainger, 1991, 1992; Segui \& Grainger, 1990; Sereno, 1991). By varying the physical and/or more abstract properties shared by prime and target, or the task (e.g., classification vs. naming), one attempts to identify the nature of the mental codes involved in target processing. In addition, by varying the amount of information that can be extracted from the prime in a given time interval (e.g., by crossing the factors prime type and prime duration), one can also examine the dynamics of the activation of these levels of representation (Arguin \& Bub, 1994, in press; Ferrand \& Grainger, 1993; Jacobs \& Grainger, 1991).

However, in all kinds of priming studies the same problem reappears. This concerns the definition of a proper control or baseline condition. It is standard practice in the letter and word recognition literature to choose a "neutral" condition as baseline. In studies of letter and word priming, blank stimuli, asterisks, or Xs are popular neutral primes. The reaction time (RT) obtained in such neutral conditions is considered the relative zero point. If RT in another condition is shorter, one speaks of facilitation; if it is longer, this is considered evidence for inhibition. Precautions have to be taken when using neutral 
primes, however, and the definition of the term neutral in a model-free, absolute way remains a problem (Jonides \& Mack, 1984).

Consider the following examples. In an alphabetic decision task (i.e., speeded letter/nonletter classification) using masked primes, a blank character has been used as a "neutral baseline" (Jacobs \& Grainger, 1991, Experiment 1). However, it can be argued that a blank prime gives the subject nothing to process and therefore is not directly comparable with other priming conditions, in which there is always something to process. Accepting this argument, which was advanced by a reviewer of our previous paper, we chose an asterisk $\left({ }^{*}\right)$ as the neutral baseline in the second experiment of our previous study. However, given the letter/nonletter classification task, it can be argued that an asterisk is not neutral in that it may bias the decision system toward a nonletter response (Arguin \& Bub, in press; Jacobs \& Grainger, 1991). The same logic can be applied to a lexical decision task in which a row of Xs or asterisks are standard neutral primes.

One way to deal with the difficulty of defining a neutral baseline is to define it within the context of a processing model that specifies its effects on target processing relative to those of nonneutral primes. This is the approach chosen by Grainger and Jacobs (1993a, 1993b, 1994) for a lexical decision task and by Arguin and Bub (in press) for an alphabetic decision task. In the present article, we propose an alternative way. We present a novel technique for evaluating facilitatory and inhibitory effects in studies of letter and word priming that uses a within-condition baseline. The technique uses a prime intensity increment method to measure the size of priming effects for each priming condition with respect to itself. In the baseline condition, prime intensity is set at a level sufficiently low to yield a null priming effect. The zero point is thus defined as the level of prime energy or availability for which no influence on target processing is observed. Prime intensity is then gradually increased (i.e., incremental priming). If RT decreases in comparison with the zero condition, this is taken as evidence that increasing the availability or information value of the prime facilitated target processing. If RT increases, we take this as evidence for inhibition of target processing. Thus, we can define any priming effect with respect to two baseline conditions: one is the minimum-intensity condition of the particular priming condition (withincondition baseline), and the other is a different priming condition (across-condition baseline).

We argue that this double-baseline approach makes measuring of priming effects more reliable and imposes stronger constraints on our interpretations of these effects. On the one hand, the within-condition baseline cannot replace theoretically motivated across-condition comparisons that aim at determining the locus of priming effects. On the other hand, as we will argue below, it is difficult to claim that a prime that yielded shorter RTs than in a given neutral baseline but did not shorten RT when becoming more and more available (i.e., in incremental priming) really facilitated target processing (see introduction to Experiment 2 below). Thus, the doublebaseline technique presented here provides doublesafety for theoretical interpretations of priming effects.

The present study extends our previous work on letter and word priming (Ferrand \& Grainger, 1993; Grainger \& Jacobs, 1991, 1993a; Jacobs \& Grainger, 1991, 1992) in that we directly compare letter and word priming effects in the alphabetic decision task and the lexical decision task while examining the dynamics of priming effects through use of the novel incremental technique. The study is mainly intended to provide a methodological contribution. Theoretical discussions are kept very brief. The reader should refer to our previous papers (Grainger \& Jacobs, 1993a, 1993b, 1994; Jacobs \& Grainger, 1991, 1992) or to the paper by Arguin and Bub (in press) for model-theoretic interpretations and simulations of priming effects in the alphabetic and lexical decision tasks.

\section{EXPERIMENT 1 Letter Priming in the Alphabetic Decision Task}

Experiment 1 examined the dynamics of letter priming effects as a function of prime intensity and prime type. The method uses a combination of the yes/no alphabetic decision task with the masked priming and the intensity increment technique.

We were mainly interested in determining the conditions under which lowercase versions of a letter facilitate classification of its uppercase version. Although facilitatory effects of nominally identical primes have been observed in a number of matching, alphabetic decision or naming task studies (Arguin \& Bub, in press; Boles, 1992; Eichelman, 1970; Jacobs \& Grainger, 1991; Posner, 1978), the effects are often small and seem to depend crucially on the exact stimulus material, task, and experimental conditions used. Thus, the stimulus onset asynchrony, the type-font, the definition of the baseline condition, and visual and/or phonological similarity relations between the prime and target sets all can play a role. These methodological issues complicate the interpretation of these priming effects that have been accounted for in terms of phonological mediation (Posner, 1978), graphemic mediation (Arguin \& Bub, in press; Besner, Coltheart, \& Davelaar, 1984), or fast visual generation (Boles, 1992).

In Experiment 1, we present the incremental priming technique as a method that, by use of a double-baseline approach, imposes stronger constraints on these theoretical interpretations.

\section{Method}

Subjects. Four well-trained subjects, all members of the experimental psychology lab in Paris, participated in this experiment. One of the subjects was the first author. All subjects had normal or corrected-to-normal vision. 
Apparatus, Stimuli, and Design. Stimulus presentation and response measurements were controlled by an Acorn-BBC Archimedes 410 workstation using a greenish monitor with a 50$\mathrm{Hz}$ refresh rate. The stimuli were taken from the type font "Mode 4" of the computer. Each character was about $0.4 \mathrm{~cm}$ high and $0.3 \mathrm{~cm}$ wide. Viewing distance was maintained constant at $50 \mathrm{~cm}$. A character subtended approximately $0.35^{\circ}$ (horizontally) and $0.45^{\circ}$ (vertically) of visual angle. Stimuli were presented at a high contrast under photopic viewing conditions. The target letters were 10 uppercase consonants (B, C, D, F, G, H, J, K, L, M), and the character foils were 10 keyboard characters $\left[\%, \mathfrak{£},:,{ }^{*},+, ?,=\right.$, ), <, > ] similar to those used by Greenberg and Vellutino (1988) and Jacobs and Grainger (1991). Target letters were presented in uppercase and preceded by four different prime types: (1) Physically identical, the prime was physically identical to the target (e.g., BB). (2) Nominally identical, the prime had the same name as the target and was presented in lowercase (e.g., bB). (3) Different, the prime was a different letter from the target and not a member of the target set (e.g., XB; 10 consonants-N, P, Q, R, S, T, V, W, X, Y-were used here). (4) Nonletter, the prime was a nonletter taken from the above pool of target foils. The character foils were preceded by either different nonletters belonging to the target foil set (nonletter/nonletter condition, e.g., $* \%$ ) or letters taken from the different prime set (e.g., $A \%$ ).

Each of the 10 target letters and character foils was presented twice in each of the four priming conditions, giving a total of 160 trials per block and subject. Four different prime intensities were used. Prime intensity was defined as the difference in intensity level between the prime and the backward mask. The Archimedes computer provided 16 stable intensity or gray steps (i.e., combinations of red, green, and blue). These were collapsed into four levels. The maximum step (16) was used for the forward and backward masks and the target stimuli. This corresponded to a maximum value of approximately $13.7 \mathrm{~cd} / \mathrm{m}^{2}$ (the background intensity of the screen was about $1.5 \mathrm{~cd} / \mathrm{m}^{2}$ ). The four different primeintensity levels were: level 1 (approximately $25 \%$ of the maximum intensity), level $2(50 \%$ of the maximum intensity), level $3(75 \%$ of the maximum intensity), and level 4 (i.e., 100\%). The prime type factor was varied within blocks, and the prime intensity factor was varied between blocks, the order being counterbalanced within each subject in a Latin square design. That is, each subject cycled through the four blocks of 160 trials four times, but in a different order across subjects. Thus, the total number of trials per subject was 2,560.

Procedure. The same procedure as the one used in the last two experiments of Jacobs and Grainger (1991) was adopted here. The sequence of events on each trial was as follows. A checkerboard mask was presented on the center of the screen for $500 \mathrm{msec}$. This was immediately replaced in the same position by the prime stim- ulus, which remained on the screen for $60 \mathrm{msec}$. The prime stimulus disappeared and was replaced by a backward mask, which remained on the screen for one frame duration $(20 \mathrm{msec})$. Immediately after, the target letter appeared in the same position and on the same line. The target remained on the screen until the subject responded by pressing one of two buttons with the index finger of either the left or the right hand to indicate if the target was a nonletter (pressing the $Z$ key on the left) or a letter (pressing the / key on the right). The subjects were asked to fixate the pattern mask each time it appeared and then to respond as quickly as possible to the target appearing one line below. The RTs were measured from target onset to subject's response.

\section{Results}

Mean RT for correct letter and nonletter classification for the different priming conditions, along with percentage of errors, is summarized in Table 1. An analysis of variance (ANOVA) using a repeated measures (prime type $\times$ prime intensity) design showed no significant error effects or interactions.

The RT results, submitted to a repeated measures ANOVA, yielded a significant main effect of prime type on RT $[F(5,15)=49.15, p<.001]$ but no reliable effect of prime intensity $[F(3,9)=0.542$, n.s. $]$. The significant interaction between the two factors $[F(5,45)=28.69$, $p<.001$ ] indicates that the effects of prime type increased with prime intensity but in different directions. For example, although increasing prime intensity shortened RT in the physically identical condition, it prolonged RT in the different condition.

Across-condition priming effects. The data in Table 1 indicate that for both letter and nonletter targets, clear across-condition priming effects were obtained for prime-intensity levels 2, 3, and 4. Averaged across these intensity levels, RT to letter targets in the physically identical condition was about $35 \mathrm{msec}$ shorter than in the nominally identical condition, $50 \mathrm{msec}$ shorter than in the different condition, and about $85 \mathrm{msec}$ shorter than in the nonletter condition. RT to nonletter targets was, on average, about $40 \mathrm{msec}$ shorter in the nonletter condition than in the letter condition.

For each of those three intensity levels, planned, orthogonal comparisons were carried out, in lieu of the overall $F$ test (Rosenthal \& Rosnow, 1985). For prime-

Table 1

Mean Correct Reaction Times (RTs, in Milliseconds) With Standard Deviations and Percentage of Errors by Prime Type and Prime Intensity for Letter and Nonletter Targets in Experiment 1

\begin{tabular}{|c|c|c|c|c|c|c|c|c|c|c|c|c|}
\hline \multirow[b]{3}{*}{ Prime Type } & \multicolumn{12}{|c|}{ Prime Intensity } \\
\hline & \multicolumn{3}{|c|}{1} & \multicolumn{3}{|c|}{2} & \multicolumn{3}{|c|}{3} & \multicolumn{3}{|c|}{4} \\
\hline & $M$ & $S D$ & $\%$ Errors & $M$ & $S D$ & $\%$ Errors & $M$ & $S D$ & $\%$ Errors & $M$ & $S D$ & $\%$ Errors \\
\hline \multicolumn{13}{|l|}{ Letter Targets } \\
\hline Physically identical & 390 & 32 & 0.7 & 353 & 23 & 0.1 & 349 & 21 & 0.2 & 346 & 20 & 0.1 \\
\hline Nominally identical & 392 & 28 & 0.9 & 385 & 24 & 0.7 & 389 & 17 & 0.6 & 388 & 19 & 0.6 \\
\hline Different & 391 & 27 & 1.3 & 395 & 25 & 1.2 & 404 & 19 & 1.1 & 405 & 25 & 1.2 \\
\hline Nonletter/letter & 394 & 31 & 1.4 & 428 & 18 & 3.1 & 438 & 19 & 2.6 & 443 & 14 & 3.4 \\
\hline \multicolumn{13}{|l|}{ Nonletter Targets } \\
\hline Letter/nonletter & 397 & 27 & 1.4 & 414 & 19 & 1.5 & 420 & 16 & 1.0 & 425 & 21 & 1.5 \\
\hline Nonletter/nonletter & 398 & 26 & 0.8 & 370 & 14 & 0.2 & 378 & 12 & 1.0 & 380 & 18 & 0.5 \\
\hline
\end{tabular}


intensity level 2 , RT to letter targets in the physically identical condition was significantly shorter than in the nominally identical condition $[F(1,3)=61.4, p<.005]$, which, in turn, gave a marginally significant difference with the different condition $[F(1,3)=5.6, p<.1]$. Mean RT in the different condition was significantly shorter than in the nonletter condition $[F(1,3)=20.51, p<$ .025]. For nonletter targets, $\mathrm{RT}$ in the nonletter condition was significantly shorter than in the letter condition $[F(1,3)=112.1, p<.005]$. For prime-intensity level 3, we obtained the following results: for letter targets, physically identical versus nominally identical $[F(1,3)=95.9$, $p<.005]$, nominally identical versus different $[F(1,3)=$ $12.6, p<.05]$, and different versus nonletter $[F(1,3)=$ $70.3, p<.005]$; for nonletter targets, nonletter versus letter $[F(1,3)=107, p<.005]$. The same was true for prime-intensity level 4: for letter targets, physically identical versus nominally identical $[F(1,3)=108.2, p<$ $.005]$, nominally identical versus different $[F(1,3)=$ $16.3, p<.05]$, different versus nonletter $[F(1,3)=86.5$, $p<.005]$; for nonletter targets, nonletter versus letter $[F(1,3)=120, p<.005]$.

Within-condition priming effects. The intensity increment technique provides a supplementary way to determine the direction and amplitude of priming effects. Taking intensity level 1 as baseline in each of the priming conditions, one simply has to see whether increasing intensity leads to a decrease or increase in RT. If RT decreases, the prime had an incremental facilitatory effect on target processing; if RT increases, it inhibited target processing. Obviously, the baseline RT has to be similar (i.e., not significantly different) in the different priming conditions for this method to work. The results of an ANOVA confirmed the absence of any significant effects of prime type for intensity level 1 .

Figure 1 gives the amount of net RT facilitation and inhibition for each priming condition as a function of prime intensity for letter (Figure 1A) and nonletter (Figure 1B) targets. The curves in Figure 1 indicate RT facilitation for letters in the physically identical condition and for nonletters in the nonletter condition, RT inhibition for letters in the nonletter condition and for nonletters in the letter condition, and small, if any, effects of prime intensity in the nominally identical and different conditions for letters.

A significant decreasing linear trend was obtained with physically identical primes for letter targets $[F(1,3)=$ $87.9, p<.005]$ and a marginally significant linear trend with nonletter primes for nonletter targets $[F(1,3)=$ $7.51, p<.1]$. For both conditions, we obtained a significant quadratic component $[F(1,3)=26.8, p<.025$, and $F(1,3)=15.2, p<.05$, respectively]. Significant increasing linear trends were obtained in the nonletter condition for letter targets $[F(1,3)=35.73, p<.01]$ and in the letter condition for nonletter targets $[F(1,3)=24.9$, $p<.025]$. No significant linear or quadratic trends were obtained for the nominally identical and different conditions. However, orthogonal contrasts for the different condition showed that RT was marginally significantly longer

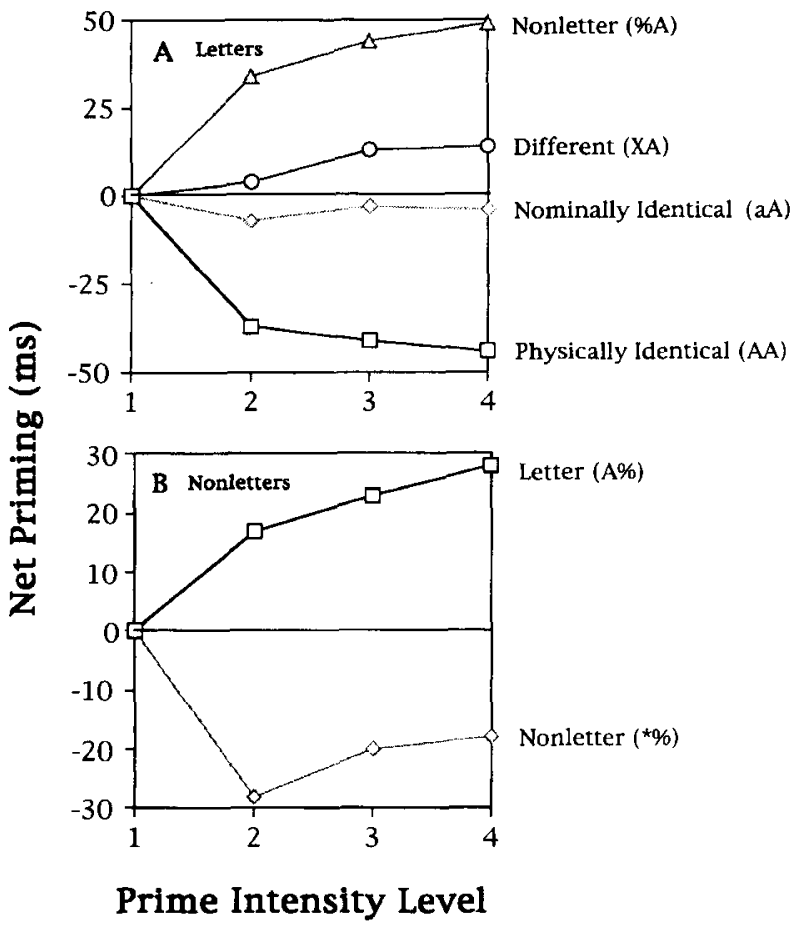

Figure 1. Net priming for letter targets (A) and nonletter targets (B) by prime type and prime-intensity level in Experiment 1. Net priming is computed as the difference (in milliseconds) between RT, in each priming condition, for intensity level 1 and RT for the other intensity levels [e.g., net4 = RT(4) - RT(1)]. Thus, positive values reflect inhibition, relative to the baseline.

for prime-intensity level 3 than for level $1[F(1,3)=9.36$, $p<.1]$.

\section{Discussion}

The results of Experiment 1 using a psychophysical approach with few subjects, many trials, and the intensity increment technique shed a new light on the interpretation of facilitation and inhibition in priming studies. The striking finding of this experiment is that RT facilitation is obtained for letters in the nominally identical condition if the different condition serves as baseline (see across-condition priming effects above), but not if a within-condition baseline is used (i.e., prime-intensity level 1). Had we run an experiment using only primeintensity level 4 , as in our previous study, we might have concluded on the basis of these results that we have clear evidence for facilitation and hence for a contribution of abstract letter detectors to processing in the alphabetic decision task (Jacobs \& Grainger, 1991).

However, the present analyses of within-condition priming effects suggest that this definition of facilitation may be misleading, since neither in the nominally identical condition nor in the different condition for letters were any significant linear trends with increasing prime intensity obtained (see Figure 1A). This raises the question of what is facilitated by a nominally identical letter prime that by definition becomes increasingly available 
to the processing system but does not alter target processing time? One could argue that a (statistically) flat priming function, as in the nominally identical condition, means that priming is already maximally present at the lowest intensity level. However, the absence of significant across-condition differences at intensity level 1 invalidates this argument.

To put stronger constraints on models of priming, we would therefore like to propose the following rule: Both significant across-condition and within-condition priming effects should be measured in theoretically crucial priming experiments and/or conditions. If only one of these two priming effects is obtained, the data are not clearly interpretable. An analogy with psychopharmacological methodology may be helpful here. If, in a psychopharmacological study, one obtains a significant drug versus placebo effect, but no dose effect (i.e., drug level 1 vs. level 2), the data are generally considered as not interpretable. We propose a similar logic for priming studies.

For example, we obtain clear RT facilitation in the physically identical condition for letter targets when either prime-intensity level 1 or the different condition serve as baseline (see Table 1 and Figure 1). For this priming condition, we can thus conclude that (1) when the prime becomes increasingly available to the processing system, it has an increasingly facilitatory effect on target processing (as shown by the significant statistical trends), and (2) target processing is easier (i.e., shortened) than in some theoretically interesting reference condition, such as the nominally identical or different conditions. This double-baseline priming effect is clearly interpretable within the framework of activation models of letter and word recognition (see introduction; Arguin \& Bub, in press; Jacobs \& Grainger, 1991).

On the other hand, according to the aforementioned rule, we should be more cautious with interpretations of the priming effects observed in the nominally identical condition, since we obtained only an across-condition priming effect. Any account of the present nominally identical, across-condition priming effect as a real facilitatory effect should also account for the fact that an increasingly available, nominally identical prime did not significantly shorten target processing time, whereas physically identical primes did. There is one such account in terms of an interactive activation type model of letter priming (Jacobs \& Grainger, 1991). Thus, one can consider that, in the physically identical condition, both feature detectors and abstract letter detectors tuned to the target are preactivated by the prime. In contrast, in the different condition, these detectors would be inhibited. However, in the nominally identical condition, one could argue that the preactivation at the level of abstract letter detectors and the inhibition at the level of feature detectors cancel each other out, because of increased preactivation of feature detectors with increasing prime intensity. Although this possibility remains to be examined by way of simulation studies, which are outside the scope of this paper, recent independent evidence sug- gests that there is no facilitatory nominally identical priming in the alphabetic decision task (Arguin \& Bub, in press). Therefore, the present significant difference in RT for the nominally identical versus different condition is best interpreted as reflecting an inhibition of target processing in the different condition, rather than a true facilitation in the nominally identical condition.

\section{EXPERIMENT 2 Word Priming in the Lexical Decision Task}

In Experiment 2, we applied the incremental priming technique to the lexical decision task, which is quite similar to the alphabetic decision task but has been more widely used to study priming effects. We were interested in reexamining standard priming effects obtained in this task by using the incremental priming technique. Thus, it was an empirical question whether the nominally identical condition (e.g., word-WORD) would yield RT facilitation at all for decision times to words (see Forster \& Davis, 1984; Grainger \& Jacobs, 1993a; Sereno, 1991) when the new definition of both across-condition and within-condition priming introduced in the present article is applied. Until now, nominally identical facilitation has always been defined with respect to another priming condition, but, as we have argued above, the demonstration of within-condition facilitation would constrain theoretical interpretations of this effect.

Suppose we obtained shorter overall lexical decision times for word targets primed by their lowercase versions (e.g., word-WORD) than for words primed by other words (shoe-WORD), and, at the same time, a flat prime-intensity function in the word-WORD condition along with an increasing function in the shoe-WORD condition. Would this result represent conclusive evidence in favor of nominally identical facilitation, and, hence, its standard interpretation that processing of the prime altered processing at some abstract representation level? Or, rather, does it establish the presence of inhibition in the shoe-WORD condition?

Clearly, a result that would be easier to interpret, at least in terms of current activation models of letter and word recognition, would be the following: a decreasing prime-intensity function in the word-WORD condition and an increasing function in the shoe-WORD condition. One could then reasonably argue that target processing at some hypothetical representation level(s) has been altered by a nominally identical prime.

On the other hand, the theoretical interpretation of the locus of priming effects requires across-condition comparisons. Thus, supposing we obtain nominally identical facilitation for word targets in terms of within-condition comparisons, we can try to answer the question about the origin of this effect by looking at whether we also obtained physically identical and nominally identical facilitation for nonwords or only physically identical facilitation. If both physically identical and nominally identical facilitation obtains for nonwords, a nonlexical, graphemic origin of nominally identical priming effects 
for both words and nonwords remains the most parsimonious interpretation. If only physically identical, and not nominally identical, facilitation is obtained for nonwords, then a lexical interpretation of nominally identical priming effects for words receives more support (Forster \& Davis, 1984).

A second issue addressed in Experiment 2 concerns the presence of response competition effects in the lexical decision task. Contrary to the alphabetic decision task in which response competition yields strong inhibitory effects in the nonletter/letter and letter conditions (Jacobs \& Grainger, 1991; see also results of Experiment 1), no evidence for the presence of such response competition in the lexical decision task has been reported. In the unrelated (different) condition using the Forster and Davis (1984) masked priming technique, it does not seem to matter whether the prime is a word or a nonword (Forster, personal communication, March 1993; Sereno, 1991). A possible methodological explanation for this discrepancy between the alphabetic decision task and the lexical decision task is that, for example, the primes used in the alphabetic decision task in both the nonletter and the letter conditions also served as targets in the corresponding physically identical conditions. If unrelated primes never serve as targets, the absence of response competition is less surprising.

To tackle this question, we used two types of primes in the different condition. Half of them also served as targets, the other half did not. If we find response competition for both types of different primes, then this suggests a global competition, independent of stimulus identity, simply due to the fact that subjects establish a stable association between word stimuli and one particular response and between nonwords and another response. In contrast, if we find response inhibition only for those primes that also served as targets, this suggests a contribution of local representations to response competition.

To summarize, in Experiment 2, we examined the effects of physically identical, nominally identical, and different primes on decision times to words and nonwords using the incremental priming technique as a supplementary analytical tool. In addition, we studied response competition effects in the lexical decision task by including a nonword priming condition for word targets and a word priming condition for nonword targets.

\section{Method}

Subjects. Eight well-trained subjects (members and students of the Cognitive Neuroscience Lab in Marseille) participated in this experiment. All subjects had normal or corrected-to-normal vision.

Apparatus, Stimuli, and Design. Stimulus presentation and response measurements were controlled by an Acorn-BBC Archimedes 410 workstation. Stimuli were presented at a high contrast under photopic viewing conditions, very similar to those of Experiment 1. The target words were 40 high-frequency words chosen from Trésor de la Langue Française (1971) and were always presented in uppercase. Their frequency range was $60-8,500$ occurrences per million, the average frequency being 1,570 . For the purpose of the lexical decision task, 40 phonologically legal nonwords (pseudowords) were constructed by changing one letter of 40 high-frequency French words (10 times in the 1st, 2nd, 3rd, and 4 th position) that were not in the target word list. No pseudohomophones were used.

Four priming conditions for word targets were used: (1) Physically identical, the prime was physically identical to the target (e.g., YEUX-YEUX). (2) Nominally identical, the prime had the same name as the target but was presented in lowercase (e.g., yeuxYEUX). (3) Different, the prime was a different high-frequency word that was not a member of the target word list and that shared no letters with the target (e.g., pris-YEUX). Half of the different primes were presented in uppercase; the other half were in lowercase. (4) Opposite, the prime is a pronounceable nonword (e.g., BOIN-YEUX). Half of the primes in the nonword condition were taken from the set of target nonwords; the other half were new ones. The nonword targets were presented in the corresponding four priming conditions: physically identical, nominally identical, different, and opposite (i.e., word).

The 40 target words were divided into four lists, matched for frequency and summed positional bigram frequency (see Grainger \& Jacobs, 1993a). Each list was used in a different priming condition for a particular level of prime intensity. Each target word was seen once at each level of prime intensity but in a different priming condition (see Grainger \& Jacobs, 1993a). Thus, one subject could see the target word YEUX at level $I$ in the physically identical condition, at level 2 in the nominally identical condition, and so on, and another subject could see the same target in a different prime intensity/prime type combination. Each of the target words and nonwords was presented once in each of the four prime type conditions, giving a total of 80 trials per block and subject (i.e., 320 experimental trials per subject). There were also 10 training trials at the beginning of each block, 5 using word targets and 5 using nonword targets. Prime type varied within blocks, and prime intensity varied between blocks, the order being counterbalanced across subjects in a Latin square design.

Procedure. Standard lexical decision procedure was adopted, combined with the fast masked priming and intensity increment technique. The sequence of events on each trial was as follows. A forward mask consisting of three rows of four \# signs (\#\#\#) was presented on the center of the screen for $500 \mathrm{msec}$. This was immediately replaced by the prime stimulus, which remained on the screen for $60 \mathrm{msec}$ and was displayed at one of four possible intensity levels, as in the procedure of Experiment 1. The prime disappeared and was replaced by a backward mask (the same as the forward mask), which remained on the screen for one frame duration $(20 \mathrm{msec})$. Immediately after, the target stimulus appeared in the same position and on the same line as the prime. The target remained on the screen until the subject responded by pressing one of two buttons with the index finger of either the left or the right hand to indicate if the target was a nonword (pressing the $Z$ key on the left) or a word (pressing the / key on the right). Mean RT was measured from target onset to subject's response. It is useful to note differences between the present masked priming technique and the one introduced by Forster and Davis (1984). First, instead of using words, we used a pattern forward mask. Second, Forster and Davis did not present a mask between the prime and the target. This prevented them from using a physically identical condition, which we wanted to include here for compatibility with Experiment 1.

\section{Results}

A repeated measures ANOVA indicated significant main effects on RT of prime type $[F(3,21)=68.07, p<$ $.001]$ and response type $[F(1,7)=66.23, p<.001]$, but no reliable effect of prime intensity $[F(3,21)=1.83$, n.s.]. The interaction between prime type and intensity was significant $[F(9,63)=27.15, p<.001]$, indicating that the effects of prime type increased with prime in- 
tensity. The interaction between prime type and response type was also significant $[F(3,21)=16.9, p<.001]$. The results are summarized in Table 2.

Across-condition priming effects. Separate analyses for word and nonword targets and for the four primeintensity levels indicated that, for the two lowest primeintensity levels, the effect of prime type was not significant. However, for the two highest prime-intensity levels, the data in Table 2 show clear across-condition priming effects.

Words. Table 2 shows that, for prime-intensity levels 3 and 4, RT to word targets primed by either physically or nominally identical words was facilitated by about $80 \mathrm{msec}$ with respect to the different condition. The latter, in turn, yielded RTs that were about $20 \mathrm{msec}$ shorter than those in the opposite condition. Planned comparisons (orthogonal contrasts) carried out between the theoretically interesting conditions confirmed that RT in the nominally identical condition was significantly shorter than in the different condition for both intensity level $3[F(1,7)=40.8, p<.001]$ and intensity level 4 $[F(1,7)=64.00, p<.001]$. Mean RT to different-primed words was significantly shorter than mean RT to words preceded by nonwords for both intensity levels $[F(1,7)=6.54, p<.05$, and $F(1,7)=11.2, p<.025$, respectively]. There was no significant difference between the two types of different primes (uppercase vs. lowercase) or the two types of nonword primes (old vs. new) for either of these two intensity levels.

Nonwords. Table 2 shows that, for nonword targets, only physically identical primes yielded RT facilitation for the two highest intensity levels: RT was about $50 \mathrm{msec}$ shorter than in the different condition. As for word targets, RT was also about $20 \mathrm{msec}$ shorter in the different condition than in the opposite condition. For prime intensity levels 3 and 4 , the effect of physically identical primes on RT was significantly facilitatory with regard to both the nominally identical condition $[F(1,7)=6.00, p<.05$, and $F(1,7)=14.35, p<.01$, respectively] and the different condition $[F(1,7)=10.76$, $p<.025$, and $F(1,7)=49.85, p<.001$, respectively]. Moreover, RT to different-primed nonwords was signif- icantly shorter than RT to nonwords primed by words for intensity level $3[F(1,7)=8.97, p<.025]$ but not for intensity level $4[F(1,7)=0.87$, n.s. $]$. No significant differences were obtained for either the two types of different primes (uppercase vs. lowercase) or the two types of word primes (old vs. new).

Within-condition priming effects. Figure 2 gives the amount of net priming obtained in the four priming conditions as a function of prime-intensity level for both words and nonwords.

Words. The curves in Figure 2A illustrate that, in the physically identical and nominally identical conditions, RT to words was gradually facilitated with increasing prime intensity, whereas it was inhibited in the different and nonword conditions. The results of trend analyses confirm this. There were significant linear decreasing trends in both the physically identical condition $[F(1,7)=59.57, p<.001]$ and the nominally identical condition $[F(1,7)=37.14, p<.001]$; whereas there was a marginally significant linear increasing trend in the different condition $[F(1,7)=4.76, p<.1]$ and a significant one in the opposite condition $[F(1,7)=8.9, p<$ .025].

Nonwords. Figure 2B shows a clear decrease in RT in the physically identical condition with increasing prime intensity and a clear inhibitory trend in the opposite condition. In the nominally identical and different conditions, the curves are similar, and no clear within-condition facilitation or inhibition appears. The results of trend analyses confirm a significant linear decreasing trend for physically identical primes $[F(1,7)=79.04, p<$ $.001]$ and a linear increasing trend for word primes $[F(1,7)=11.5, p<.025]$. The linear decreasing trend in the nominally identical condition was marginally significant $[F(1,7)=4.4, p<.1]$, and only a quadratic increasing trend reached significance in the different condition $[F(1,7)=8.9, p<.025]$.

Error rates. Statistical analysis of the error rates showed no significant main effects, but a significant interaction of prime type $\times$ response type $[F(3,21)=$ $4.41, p<.025]$. This interaction reflects the fact that, for word targets, the subjects made fewer errors in the phys-

Table 2

Mean Correct Reaction Times (RTs, in Milliseconds) With Standard Deviations and Percentage of Errors by Prime Type and Prime Intensity for Word and Nonword Targets in Experiment 2

\begin{tabular}{|c|c|c|c|c|c|c|c|c|c|c|c|c|}
\hline \multirow[b]{3}{*}{ Prime Type } & \multicolumn{12}{|c|}{ Prime Intensity } \\
\hline & \multicolumn{3}{|c|}{1} & \multicolumn{3}{|c|}{2} & \multicolumn{3}{|c|}{3} & \multicolumn{3}{|c|}{4} \\
\hline & $M$ & $S D$ & $\%$ Errors & $M$ & $S D$ & $\%$ Errors & $M$ & $S D$ & $\%$ Errors & $M$ & $S D$ & $\%$ Errors \\
\hline \multicolumn{13}{|l|}{ Word Targets } \\
\hline Physically identical & 462 & 24 & 10 & 450 & 24 & 6.2 & 411 & 12 & 3.7 & 399 & 11 & 7.5 \\
\hline Nominally identical & 461 & 36 & 7.5 & 459 & 17 & 7.5 & 407 & 23 & 1.2 & 405 & 15 & 0 \\
\hline Different & 465 & 43 & 8.7 & 456 & 26 & 13 & 471 & 37 & 12.5 & 493 & 34 & 12.5 \\
\hline Nonword & 459 & 40 & 8.7 & 473 & 23 & 7.5 & 493 & 37 & 11.2 & 513 & 30 & 22.2 \\
\hline \multicolumn{13}{|l|}{ Nonword Targets } \\
\hline Physically identical & 496 & 24 & 8.7 & 477 & 22 & 11.2 & 449 & 13 & 8.7 & 436 & 16 & 6.2 \\
\hline Nominally identical & 498 & 32 & 8.7 & 486 & 24 & 12.5 & 490 & 23 & 16.2 & 488 & 26 & 13.7 \\
\hline Different & 495 & 25 & 10 & 487 & 19 & 17.5 & 482 & 21 & 18.5 & 498 & 24 & 5 \\
\hline Word & 489 & 37 & 20 & 484 & 21 & 4.5 & 507 & 20 & 9.5 & 515 & 25 & 14.5 \\
\hline
\end{tabular}






Figure 2. Net priming for word targets (A) and nonword targets (B) by prime type and prime-intensity level in Experiment 2 . Net priming is computed as the difference (in milliseconds) between RT, in each priming condition, for intensity level 1 and $R T$ for the other intensity levels [e.g., net4 = RT(4) - RT(1)]. Thus, positive values reflect inhibition, relative to the baseline.

ically identical and nominally identical conditions $(6.85 \%$ and $4.05 \%$, respectively) than in the different and nonword conditions ( $11.6 \%$ and $12.4 \%$, respectively); however, for nonword targets, error rate was lowest for the physically identical condition $(8.7 \%)$, but no differences were observed for the three other priming conditions $(12.77 \%, 12.75 \%$, and $12.12 \%$; see Table 2$)$.

\section{Discussion}

The results of Experiment 2 demonstrate that words are classified more rapidly as words when they are preceded by either a physically or a nominally identical stimulus. This facilitatory priming effect, which is about equal in both conditions, is obtained in terms of both within-condition and across-condition comparisons (i.e., nominally identical vs. different primes). We can thus be reasonably confident that subjects use some abstract information in the lexical decision task either at the sublexical level of letter types (Humphreys et al., 1990; Sereno, 1991) or at higher lexical (Forster \& Davis, 1984) or decision levels (Grainger \& Jacobs, 1993b, 1994).

The finding of a significant linear decreasing trend in the nominally identical condition for words, in contrast to the increasing trend obtained in the different condition, suggests that the reason for the difference between these two priming conditions is not an absence of preactivation of abstract processing units in the different con- dition but an active inhibition of these. With increasing prime intensity, processing units that are incompatible with the subsequent target are increasingly activated and thus inhibit target processing (e.g., via inhibitory connections at the lexical or response levels; Coltheart \& Rastle, 1994; McClelland \& Rumelhart, 1981; Grainger \& Jacobs, 1993a, 1993b, 1994; Jacobs \& Grainger, 1991, 1992, 1994).

We also obtained significant RT facilitation for nonwords primed with physically identical stimuli in both the within- and the across-condition comparisons. For nominally identical primes, the adequate across-condition comparison (nominally identical vs. different) yielded no facilitatory effect, although a marginally significant trend was observed in the within-condition comparisons. Given the fact that the curves for the nominally identical condition and the different condition overlap almost perfectly, we prefer the conservative view that there is no firm evidence in our data for the presence of nominally identical facilitation with nonwords in the lexical decision task. This view agrees with our previous results based on acrosscondition comparisons (Grainger \& Jacobs, 1993a).

Note that although RTs to nonwords are shorter in the nominally identical condition than in the opposite condition, this is not an adequate across-condition comparison because of a possible response competition effect in the opposite condition. Indeed, we find evidence for response priming/competition effects in the lexical decision task. When words are preceded either by different words or by nonwords, lexical decision times are inhibited in terms of within-condition comparisons--that is, the more the prime becomes available, the longer are decision latencies. However, there is a difference here that is predicted by the results obtained in the alphabetic decision task of Experiment 1 (see also Jacobs \& Grainger, 1991). Mean RT to words preceded by nonwords is clearly more inhibited than mean RT to words primed by different words, as revealed by the across-condition comparisons. This inhibitory effect was independent of whether the nonwords served as targets or not. Thus, no evidence is obtained that there is a contribution of the (pre)activation of local representations to the additional inhibition caused by nonword primes. A straightforward parsimonious interpretation is that the sharing of the response code between prime and target in the different condition leads to less inhibition than when prime and target have opposed response codes (see Jacobs \& Grainger, 1991).

\section{GENERAL DISCUSSION}

The findings reported in this article demonstrate that target processing in speeded letter and word classification depends on prime type and prime intensity. The argument we develop in this paper, via the incremental priming technique, is that the standard interpretation of priming effects (e.g., in terms of activation models of letter and word recognition) is better constrained by a double-baseline approach than when only across-condition comparisons are made. On the other hand, within-condition 
comparisons cannot replace the theoretical value of motivated across-condition comparisons, because they do not inform us about hypothetical loci of priming effects. Hence, for theoretically crucial priming experiments, we propose the rule that double-baseline priming effects should be obtained for the data to be considered interpretable.

As with any novel experimental technique, several caveats must be made. One concerns the possibility of alertness effects. With increasing prime intensity, subjects could become increasingly alert. This increasing alertness could influence RTs over and above the priming effects that the experimenter is interested in. However, if significant across-condition effects that are compatible with the observed within-condition effects are obtained, the alertness argument should not matter. For instance, it is difficult to see how alertness effects could explain the finding of Experiment 2 that, for word targets, physically identical and nominally identical primes yielded overlapping decreasing within-condition functions, whereas different primes yielded an increasing function and nonword primes yielded an even steeper increasing function. Any alertness hypothesis would have to explain how different degrees of alertness are obtained for different prime types and for different target types (i.e., words and nonwords).

Another methodological concern may be forward masking. One could worry that increasing prime-intensity levels cause increasing degrees of forward masking. However, the fact that we used a backward mask between prime and target counters this argument. It is also hard to see how increased forward masking could yield RT facilitation with increasing prime intensity. In addition, it is difficult to see how a forward masking hypothesis could explain the differential priming effects discussed above in relation to the alertness hypothesis. For example, why should nonword primes for word targets yield stronger forward masking than different primes in the lexical decision task, as suggested by a steeper primeintensity function? Finally, one could even argue that if some nonspecific (inhibitory) forward masking effects were present in our experiments, they might have been canceled out by some nonspecific (facilitatory) alertness effects.

Thus, given that our data provide no evidence in favor of the alertness or forward masking hypotheses, we propose that a prime that becomes increasingly available to the processing system (i.e., with increasing prime intensity) but does not alter RT cannot reasonably be said to facilitate target processing, even if RT measured in this priming condition is shorter than in a given control condition. If, in addition, RT in the control condition increases with prime intensity, this suggests that what might have been interpreted as a facilitatory effect-if only a single prime intensity condition were used - is much more likely to be the result of inhibition of target processing in the control condition (see Discussion section of Experiment 1). In this respect, use of a doublebaseline, as provided by the incremental priming tech- nique, and a double-priming effect rule, as suggested above, should help prevent researchers from: drawing premature conclusions.

\section{REFERENCES}

Arguin, M., \& Bub, D. N. (1994). Pure alexia: Attempted rehabilitation and its implications for interpretation of the deficit. Brain \& Language, 47, 233-268.

ARGUIN, M., \& BUB, D. N. (in press). Letter priming and decision processes in classification and identification tasks. Journal of Experimental Psychology: Human Perception \& Performance.

Besner, D., Coltheart, M., \& Davelaar, E. (1984). Basic processes in reading: Computation of abstract letter identities. Canadian Journal of Psychology, 38, 126-134.

Boles, D. B. (1992). Fast visual generation: Its nature and chronometrics. Perception \& Psychophysics, 51, 239-246.

Carr, T. H. (1986). Perceiving visual language. In K. R. Boff, L. Kaufman, \& J. P. Thomas (Eds.), Handbook of perception and human performance (pp. 29.1-29.82). New York: Wiley.

Coltheart, M., \& Rastle, K. (1994). Serial processing in reading aloud: Evidence for dual-route models of reading. Journal of Experimental Psychology: Human Perception \& Performance, 20, 1197-1211.

EiCHELMAN, W. H. (1970). Stimulus and response repetition effects for naming letters at two response-stimulus intervals. Perception \& Psychophysics, 7, 94-96.

Ferrand, L., \& Grainger, J. (1992). Phonology and orthography in visual word recognition: Evidence from masked nonword priming. Quarterly Journal of Experimental Psychology, 33A, 325-350.

FERrAND, L., \& GRAINGER, J. (1993). The time course of orthographic and phonological code activation in the early phases of visual word recognition. Bulletin of the Psychonomic Society, 31, 119-122.

Forster, K. I., \& DAVIS, C. (1984). Repetition priming and frequency attenuation in lexical access. Journal of Experimental Psychology. Learning, Memory, \& Cognition, 10, 680-690.

Grainger, J., \& Ferrand, L. (1994). Phonology and orthography in visual word recognition: Effects of masked homophone primes. Journal of Memory \& Language, 33, 218-233.

Grainger, J., \& JaCoBS, A. M. (1991). Masked constituent letter nriming in an alphabetic decision task. European Journal of Cognitive Psychology, 3, 413-434.

Grainger, J., \& JACOBs, A. M. (1993a). Masked partial-word priming in visual word recognition: Effects of positional letter frequency. Journal of Experimental Psychology: Human Perception \& Performance, 19, $951-964$.

Grainger, J., \& JACOBS, A. M. (1993b, November). Modeling neighborhood effects in lexical decision. Paper presented at the meeting of the Psychonomic Society, Washington, DC.

Grainger, J., \& JACOBS, A. M. (1994). A three-process model of lexical decision and word recognition. Paper submitted for publication.

GreenberG, S. N., \& Vellutino, F. R. (1988). Evidence for processing of constituent single- and multiletter codes: Support for multilevel coding in word perception. Memory \& Cognition, 16, 54-63.

Humphreys, G. W., EvetT, L. J., \& Quinlan, P. T. (1990). Orthographic processing in visual word identification. Cognitive Psychology, 22, 517-560.

JACOBS, A. M., \& GRAinger, J. (1991). Automatic letter priming in an alphabetic decision task. Perception \& Psychophysics, 49, 43-52.

JaCOBS, A. M., \& Grainger, J. (1992). Testing a semi-stochastic variant of the interactive activation model in different word recognition experiments. Journal of Experimental Psychology: Human Perception \& Performance, 18, 1174-1188.

Jacobs, A. M., \& Grainger, J. (1994). Models of visual word recognition: Sampling the state of the art. Journal of Experimental Psychology: Human Perception \& Performance, 20, $1311-1334$.

Jacobs, A. M., Grainger, J., \& Ferrand, L. (1994, November). Facilitation and inhibition in letter and word recognition: The incremental priming technique. Poster presented at the meeting of the Psychonomic Society, St. Louis. 
JONIDES, J., \& MACK, R. (1984). On the costs and benefit of cost and benefit. Psychological Bulletin, 96, 29-44.

MCClelland, J. L., \& Rumel hart, D. E. (1981). An interactive activation model of context effects in letter perception: Part I. An account of basic findings. Psychological Review, 88, 375-407.

PaAp, K., Newsome, S. L., McDonald, J. E., \& Schvaneveldt, R. W. (1982). An activation-verification model for letter and word recognition: The word superiority effect. Psychological Review, 89, 573594.

PoSNER, M. K. (1978). Chronometric explorations of the mind. Hillsdale, NJ: Erlbaum.

PosNer, M. K., \& CARR, T. H. (1992). Lexical access and the brain: Anatomical constraints on cognitive models of word recognition. American Journal of Psychology, 105, 1-26.

Rosenthal, R., \& Rosnow, R. L. (1985). Contrast analysis: Focused comparisons in the analysis of variance. New York: Cambridge University Press.
Segui, J., \& Grainger, J. (1990). Priming word recognition with orthographic neighbors: Effects of relative prime-target frequency. Journal of Experimental Psychology: Human Perception \& Performance, 16, 65-76.

Selfridge, O. G., \& Neisser, U. (1960). Pattern recognition by machine. Scientific American, 203, 60-68.

Sereno, J. A. (1991). Graphemic, associative, and syntactic priming effects at a brief stimulus onset asynchrony in lexical decision and naming. Journal of Experimental Psychology: Learning, Memory, \& Cognition, 17, 459-477.

Trésor de la Langue Française [French language frequency counts] (1971). Nancy, France: Centre National de la Recherche Scientifique.

(Manuscript received August 1, 1994; revision accepted for publication May 2, 1995.) 